\title{
Heat transfer and entropy generation analysis in a horizontal channel filled with a permeable medium in the presence of aligned magnetic field and temperature gradient heat source
}

\author{
K. S. Balamurugan ${ }^{1}$ (D) N. Udaya Bhaskara Varma² $\cdot$ J. L. Ramaprasad ${ }^{3}$
}

Received: 25 May 2020 / Accepted: 16 February 2021 / Published online: 25 February 2021

(c) The Author(s) $2021 \quad$ OPEN

\begin{abstract}
The current investigation is concerned with heat transfer and entropy generation analysis in a horizontal channel brimming with porous medium in the existence of aligned magnetic field, viscous and joules dissipation and temperature gradient heat source. The boundary conditions are treated as constant values for velocity and temperature at lower and upper walls. An explicit solution of governing equations has been attained in closed system. The repercussions of pertinent parameters on the fluid velocity, temperature, entropy generation and Bejan number are conferred and scrutinized through graphs in detail. Additionally the expressions for shear stress and the rate of heat transfer coefficients at the channel walls are derived and results obtained are physically interpreted through tables. From the conquered results, it is addressed that Brinkman number Br enhances boundary layer thickness. Entropy generation increases with intensifying values of $M$, aligned angle $\phi$, temperature gradient heat source parameter $Q$, characteristic temperature ration $\omega$ and permeability parameter $\mathrm{K}$. The shear stress is same at both the lower and upper walls.
\end{abstract}

Keywords Heat transfer · Entropy generation · Aligned magnetic · Temperature gradient · Bejan number

\begin{tabular}{|c|c|c|c|}
\hline \multicolumn{2}{|c|}{ List of symbols } & Ns & Entropy generation number \\
\hline \multicolumn{4}{|c|}{$(u, v) \quad$ Velocity components in $(x, y)$ directions $\left(\mathrm{m} \mathrm{s}^{-1}\right)$} \\
\hline \multirow{2}{*}{\multicolumn{4}{|c|}{$\begin{array}{l}\text { Magnetic coefficient }\left(\mathrm{A} \mathrm{m}^{-1}\right) \\
\text { Thermal conductivity }\left(\mathrm{W} \mathrm{m}^{-1} \mathrm{~K}^{-1}\right)\end{array}$}} \\
\hline & Thermal conductivity $\left(\mathrm{W} \mathrm{m}^{-1} \mathrm{~K}^{-1}\right)$ & & \\
\hline M & Magnetic parameter & \multicolumn{2}{|c|}{ Greek symbols } \\
\hline$\left(T_{1}, T_{2}\right)$ & Temperatures at lower and upper boundary & $\phi$ & Aligned magnetic field parameter \\
\hline$Q$ & Temperature gradient heat source parameter & $\rho$ & \\
\hline$B r$ & Brinkman number & $\gamma^{*}$ & Stephan-Boltzmann constant $\left(\mathrm{W} \mathrm{m}^{-2} \mathrm{k}^{-4}\right)$ \\
\hline $\mathrm{Be}$ & Bejan number & $\theta$ & Fluid temperature $(K)$ \\
\hline$E_{G}$ & Volumetric rate of entropy generation & $\omega$ & Characteristic temperature ratio \\
\hline$\tau$ & Wall shear stress & \multicolumn{2}{|c|}{ Subscript } \\
\hline$H$ & Width of the channel (m) & * & Dimensional parameters \\
\hline K & Permeability parameter & & \\
\hline$\mu$ & Dynamic viscosity $\left(\mathrm{m}^{2} \mathrm{~s}^{-1}\right)$ & & \\
\hline$N$ & Radiation parameter & & \\
\hline$\sigma$ & Electric conductivity $\left(\mathrm{Wk}^{-1} \mathrm{~m}^{-1}\right)$ & & \\
\hline
\end{tabular}

K. S. Balamurugan, muruganbalaks@gmail.com; N. Udaya Bhaskara Varma, varma9866157033@gmail.com; J. L. Ramaprasad, jlrprasad@gmail.com | ${ }^{1}$ Department of Mathematics, RVR \& JC College of Engineering, Guntur, Andhra Pradesh 522019, India. ${ }^{2}$ Department of Basic Sciences and Humanities, DNR College of Engineering and Technology, Bhimavaram, Andhra Pradesh 534202, India. ${ }^{3}$ Department of Mathematics, PB Siddhartha College of Arts and Science, Vijayawada, Andhra Pradesh 520010, India. 


\section{Introduction}

Magnetohydrodynamics is a main branch of fluid dynamics. It is concerned with the interaction of electrically conducting fluids and electromagnetic fluids. When a conducting fluid transports through a magnetic field, an electric field, ergo current may be induced and, in turn the current combine with the magnetic field to generate a body force. Magnetohydrodynamics interactions transpire both in environment and in man-made appliances. Magnetohydrodynamics flow ensues in the earth, ionosphere, sun, stars and atmosphere. In the research laboratory bounteous innovative devices have been invented which exploit the magnetohydrodynamics interaction precisely such as propulsion units and power generators or which implicate fluid-electromagnetic field connections, like electron beam dynamics, travelling wave tunnels, electrical exonerations and abounding others.

The investigation of heat relocation and fluid flow in a penetrable channel have secured perceptible responsiveness throughout the last many decades owed to their applicability in an extensive assortment of biological and engineering like water, geophysical science irrigation and cesspit issues and also in captivation and percolation method in chemical engineering. Temperature gradients are imperative in the meteorological sciences like climatology, meteorology and allied fields. The solar light captivation proximate the planetary surfaces will proliferation the temperature gradient. The temperature gradient may amendment significantly in time, as an outcome of diurnal or seasonal warming and cooling. For illustration in the day the temperature at ground stratum could also be frost while its radiator up in the air, since the day moves over to night time the temperature valor drip briskly while at another regions on the land sojourn cooler or warmer at an commensurate ascent. This will ensue on the west coast of the United States occasionally.

It is discerned that everywhere the irreversible processes in that time equilibrium is wrecked. The disparity within reversible and irreversible process was first imported in thermodynamics by the theory of entropy. Entropy generation denigration analysis are valuable for clinching the optimal thermic methods in industrial and scientific arenas like voltaic refrigerating, warmth exchangers, geothermal structures. The generation of entropy is exactly combined by thermodynamic irreversibility. In scheming thermal system, the focus on energy usage and the entropy generation has evolve into one of the prime intents. Bejan [1] reported the potent method of reckoning entropy generation through temperature and fluid stream in heat transfer. Denigration of entropy generation is a technique for both designing and reinforcing of power structures that is given by Bejan [2]. In prior investigations associated to the natural transmission, merely the first rule of thermodynamics was applied. Though, the mode of entropy generation blends almost substantial parameters of heat deportation, thermo dynamics \& fluid dynamics. Principles and operations of entropy generation are given by Rosen [3], Narusawa [4]. Ko and Cheng [5] reported the impacts of heat transmission and entropy generation in a wavy conduit through numerical techniques. Mojtaba Aghajani Delavar and Mehdi Hedayatpour [6] investigated entropy generation by utilizing Boltzmann model in a conduit with heat producing chunk porosity. Makinde and Samuel Eigunjobi [7] applied thermo dynamics laws to analyze entropy in a duct. Tirivanhu Chinyoka and Makinde [8] explored the result of entropy generation rate and Navier slip on convective cooling flow in a permeable channel. Sanatan Dasa and Rabindra Nath Jana [9] examined in a horizontal conduit the Naver slip property on entropy generation of viscous stream by means of stable pressure gradient. Anthony Rotimi Hassan, Jacob Abiodun Gbadeyan [10] portrayed the brunt of inner warmth generation on entropy in an exothermic MHD flow by aid of Arrhenius kinetics. In this article the solutions of governing Partial differential equations are procured by adopting perturbation technique. Hatami et al. [11] studied instinctive convection heat relocation of nano-fluids in a rounded wavy-cavity by finite element technique. Sukumar and Varma [12] explored the prominence of entropy generation and viscid dissipation with temperature dependent heat source on magnetohydrodynamic generalized horizontal channel with absorbent base. Suvanjan Bhattacharyya et al. [13] analyzed the entropy as well as the enhancement of heat transmission in a wavy channel where constant temperature walls using water $\left(\mathrm{H}_{2} \mathrm{O}\right)$ as a fluid. Rashidi et al. [14] analyzed the Brownian motion of flowing non-Newtonian (third grade) with magnetic arena. To solve the non-dimensional entropy equations authors applied method of optimal homotopy analysis (OHA). Baag et al. [15] conferred the impact of entropy generation through second law of thermo dynamics to Walters $B$ liquid over stretching surface in the manifestation of both Darcy and Joule dissipation. To solve the equations of boundary layer authors exploited analytical method Kummer's function. Abiodun and Thomas [16] examined the impacts of irreversibility ratio as well as entropy cohort on steady viscid fluid flow in a vertical absorbent conduit. Wenhui Tanga et al. [17] presented geometry with dual sinusoidal wavy walls of different phase aberrations for natural convection heat transfer. Saman Rashidi et al. $[18,19]$ presented the applications 
of magnetohydrodynamics in heat transfer and nano fluids. Srinivasacharya and Hima Bindu [20] presented entropy generation and Bejan number of micro rotation polar flow fluid in a concentric annulus by means of the external cylinder with fixed velocity. In this investigation utilized quasi linearization technique to solve the equations. Sudhakar and Balamurugan [21] investigated the repercussions of (Navier) slip and entropy generation between steep parallel plates with drag/inoculation. Balamurugan et al. [22] explored the reputation of entropy generation in stable Couette flow confined by a permeable bed in the manifestation of aligned magnetic arena, viscous dissipation, thermal radiation and joules dissipation. Lalrinpuia Tlau and Surender Ontela [23] explored the impact of entropy generation for $\mathrm{Cu}$-water (nanofluid) flow in a conduit with Navier slip. PK Reddy and R Murthy [24] studied entropy generation with Bejan number in a rectangular channel with drag walls and perpetual temperature and torridity flux. Rahila Naz et al. [25] examined entropy generation in Williamson nanofluid with gyrotactic microorganisms along with a cylinder with engrossment of tending angle, Brownian motion and viscous dissipation effects.

In all the above cited articles, it is discerned that the authors are not considered temperature gradient heat source which is practically significant and boundary conditions $u^{*}=-u_{0}$ on the lower boundary and $u^{*}=u_{0}$ on the upper boundary. Therefore, this paper is apprehensive with the impacts of entropy generation as well as temperature gradient heat source on steady Couette flow with both plates lower and upper move with a uniform velocity by the means of aligned magnetic field, thermal emission, viscous dissipation and joules dissipation. An explicit solution of governing equations has been attained in closed system. The repercussions of the physical parameters on the fluid velocity, temperature, entropy generation and Bejan number are accomplished graphically and discussed in detail. Also the shear stress and heat transfer rate at the channel surfaces are derived and discussed their behavior through tables.

\section{Mathematical formulation}

Contemplate the viscous incompressible fluid confined by two endless horizontal parallel plates alienated by a width $\mathrm{H}$. The lower and higher plates move with a uniform velocity in the direction of fluid flow. A constant aligned magnetic arena of forte $B$ at an angle $\phi$ is applied in the transverse direction of fluid flow. A Cartesian coordinate system is selected with $x$-axis on the lower moving plate and the $y$-axis at right angles to the plates (Fig. 1). It is assumed that.

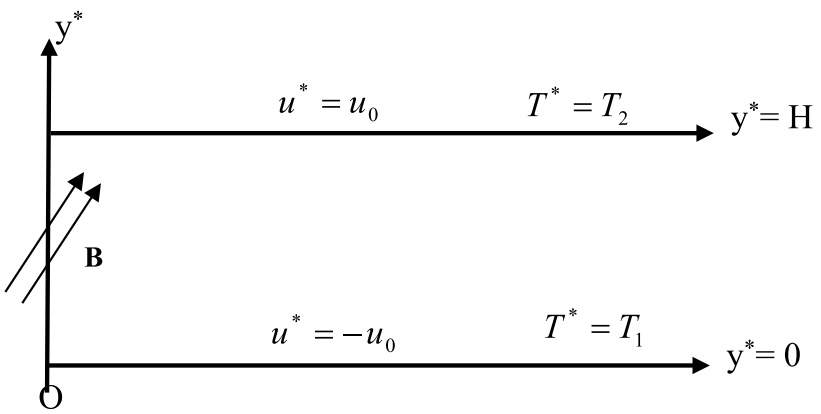

Fig. 1 Scheme diagram of the flow model

- The channel is long enough in the $x$-direction so that complete physical variables are freelance of $x$ (except pressure).

- The flow is totally developed hydro dynamically and thermally.

- Pressure and buoyancy forces as well as induced magnetic and electric fields are neglected.

- The temperatures of the lower and upper plates are $T_{1}$ and $T_{2}$ respectively, where $T_{2}>T_{1}$.

- Viscous dissipation, joules dissipation and temperature gradient heat source possessions are taken into account.

- In the equation of energy the radiative heat flux is meant to adapt Rosseland approximation.

The main momentum equation and energy equation for a steady stream of viscous incompressible fluid are conferred based on the previous studies [12].

$\mu \frac{\partial^{2} u^{*}}{\partial y^{*^{2}}}-\sigma B^{2} \sin ^{2} \phi u^{*}-\frac{\mu}{K^{*}} u^{*}=0$

$k \frac{\partial^{2} T^{*}}{\partial y^{*^{2}}}+\mu\left(\frac{\partial u^{*}}{\partial y^{*}}\right)^{2}+\sigma B^{2} \sin ^{2} \phi u^{*^{2}}-\frac{\partial q_{r}^{*}}{\partial y^{*}}+Q^{*} \frac{\partial}{\partial y^{*}}\left(T^{*}-T_{1}\right)=0$

The boundary conditions are considered as constant values for velocity and temperature for lower and upper walls, thus

$y^{*}=0: \quad u^{*}=-u_{0}, \quad T^{*}=T \quad$ On the lower boundary

$y^{*}=H: u^{*}=u_{0}, \quad T^{*}=T_{2} \quad$ On the upper boundary

where $\mu$ is forceful viscosity, $k$ is thermic conductivity, $K^{*}$ is absorptivity, $T_{1}$ is the temperature of the lower frontier, $T_{2}$ is the temperature of the upper frontier, $H$ is the distance of the channel., $B$ is magnetic field force, $\sigma$ is the electrical conductivity, $Q^{*}$ is temperature gradient heat source parameter and $\phi$ is the aligned angle. The radiation heat flux $q_{r}$ is accustomed by 
$q_{r}=\frac{-4 \gamma^{*}}{3 \alpha^{*}} \frac{\partial T^{* 4}}{\partial y^{*}}$

where $\gamma^{*}, \alpha^{*}$ are constants stands for Stephan-Boltzmann and mean immersion respectively. The temperature distinction within the fluid is sufficiently tiny thus $T^{* 4}$ expanded in a Taylor series about the free torrent temperature $T_{1}$ so that after omitting higher order terms

$T^{* 4} \cong 4 T_{1}^{3} T-3 T_{1}^{4}$

The related non- dimensional quantities are

$u=\frac{u^{*}}{u_{0}}, \theta=\frac{T^{*}-T_{1}}{T_{2}-T_{1}}, y=\frac{y^{*}}{H}, K=\frac{K^{*}}{H^{2}}$

In view of Eqs. (4-6) the Eqs. (1) and (2) become

$\frac{d^{2} u}{d y^{2}}-M^{2}\left(\sin ^{2} \phi\right) u+\frac{1}{K} u=0$

$\left(1+\frac{4 N}{3}\right) \frac{d^{2} \theta}{d y^{2}}+B r\left(\frac{d u}{d y}\right)^{2}+B r M^{2}\left(\sin ^{2} \phi\right) u^{2}+Q \frac{d \theta}{d y}=0$

The subsequent conditions on the lower and upper boundary are

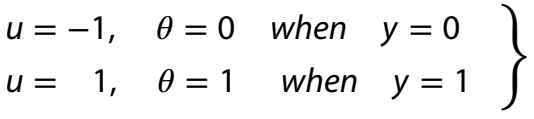

where $M^{2}=\frac{\sigma B_{0}^{2} H^{2}}{\mu}$ is Hartmann number, $B r=\frac{\mu u_{0}^{2}}{k\left(T_{2}-T_{1}\right)}$ is Brinkman number, $Q=\frac{H}{k} Q^{*}$ is temperature gradient heat source parameter $(Q>0)$ and $N=\frac{4 \gamma^{*} T_{1}^{3}}{\alpha^{*} k}$ is emission parameter.

\subsection{Entropy generation}

Entropy analysis could be a medium to quantify the thermodynamics in any fluid flow procedure. The $1^{\text {st }}$ law of thermodynamics is an interpretation principle of the conservation of energy. Thermodynamics $2^{\text {nd }}$ law states that each real procedure is irreversible. As entropy generation takes place, the eminence of energy during a field flow manner are diminish the entropy generation with in the fluid, volume entropy generation in engineering systems rescinds existing work and then diminishes its productivity. Numerous studies are accessible in live the causes of irreversibility in workings and procedures. The entropy generation rate, characteristic entropy generation rate and characteristic temperature ratio relation are given by [12]
$E_{G_{0}}=\frac{k\left(T_{2}-T_{1}\right)^{2}}{H^{2} T_{1}^{2}}$

$\omega=\frac{T_{1}}{T_{2}-T_{1}}$

Entropy generation number is

$$
\begin{aligned}
N s & =\frac{E_{G}}{E_{G_{0}}}=\left(1+\frac{4}{3} N\right)\left(\frac{d \theta}{d y}\right)^{2}+B r \omega\left[\left(\frac{d u}{d y}\right)^{2}+\left(M^{2} \sin ^{2} \phi\right) u^{2}\right] \\
& =H T I+F F I
\end{aligned}
$$

where $H T I=\left(1+\frac{4}{3} N\right)\left(\frac{d \theta}{d y}\right)^{2}$ is the heat transferal irreversibility $F F I=B r \omega\left[\left(\frac{d u}{d y}\right)^{2}+\left(M^{2} \sin ^{2} \phi\right) u^{2}\right]$ is the fluid friction irreversibility

The Bejan number Be is the appropriate irreversibility parameter and is well-defined as

$B e=\frac{H T I}{N_{s}}$

The Bejan number range concerning 0 and 1 . Where ever $B e=1$ is that the limit, at that heat transfer irreversibility dominates, $B e=0$ is that the limit at that fluid friction irreversibility dominates and $B e=1 / 2$ implies that both of them contribute equally.

\section{Solution of the problem}

To explain the entropy generation \& Bejan number on Couette flow with aligned magnetic in the manifestation of thermal radiation, temperature dependent heat source influence, dissipations (viscous and joules), it is requisite regimes of velocity and temperature. The governing equations are determined by systematic technique and it is solved by employing (7), (8) and (9). Initially, solution of the equation of momentum (7) is obtained which is used to solve equation of energy (8).

Explication of the reckonings (7) and (8) subjected to the periphery conditions (9) are attained as follows

$$
\begin{aligned}
& u=c_{1} e^{M_{1} y}+c_{2} e^{-M_{1} y} \\
& \theta=c_{3}+c_{4} e^{-\frac{Q}{B} y}+c_{5} e^{2 M_{1} y}+c_{6} e^{-2 M_{1} y}+c_{7}
\end{aligned}
$$

$E_{G}=\frac{k}{T_{1}^{2}}\left[\left(\frac{d T^{*}}{d y^{*}}\right)^{2}+\frac{16 \gamma^{*} T_{1}^{3}}{3 k \alpha^{*}}\left(\frac{d T^{*}}{d y^{*}}\right)^{2}\right]+\frac{\mu}{T_{1}}\left(\frac{d u^{*}}{d y^{*}}\right)^{2}+\frac{\sigma B^{2} \sin ^{2} \varphi}{T_{1}} u^{*^{2}}$ 


$$
\begin{aligned}
N s= & \left(1+\frac{4}{3} N\right)\left(c_{4}\left(-\frac{Q}{B}\right) e^{-\frac{Q}{B} y}+2 M_{1} c_{5} e^{2 M_{1} y}-2 M_{1} c_{6} e^{-2 M_{1} y}+c_{7}\right)^{2} \\
& +B r \omega\left[c_{1}^{2} e^{2 M_{1} y}\left(2 M_{1}^{2}\right)+c_{2}^{2} e^{-2 M_{1} y}\left(2 M_{1}^{2}\right)\right]
\end{aligned}
$$

\subsection{Skin friction}

$$
\begin{aligned}
& \tau=\left(\frac{\partial u}{\partial y}\right)_{y=0, \quad y=1} \\
& \tau_{y=0}=c_{1} M_{1}-c_{2} M_{1} \\
& \tau_{y=1}=c_{1} M_{1} e^{M_{1}}-c_{2} M_{1} e^{-M_{1}}
\end{aligned}
$$

\subsection{Nusselt Number}

$$
\begin{aligned}
& N u=\left(-\frac{\partial \theta}{\partial y}\right)_{y=0, y=1} \\
& N u_{y=0}=\frac{Q}{B} c_{4}-2 M_{1} c_{5}+2 M_{1} c_{6}+c_{7} \\
& N u_{y=1}=\frac{Q}{B} c_{4} e^{-\frac{Q}{B}}-2 M_{1} c_{5} e^{2 M_{1}}+2 M_{1} c_{6} e^{-2 M_{1}}
\end{aligned}
$$

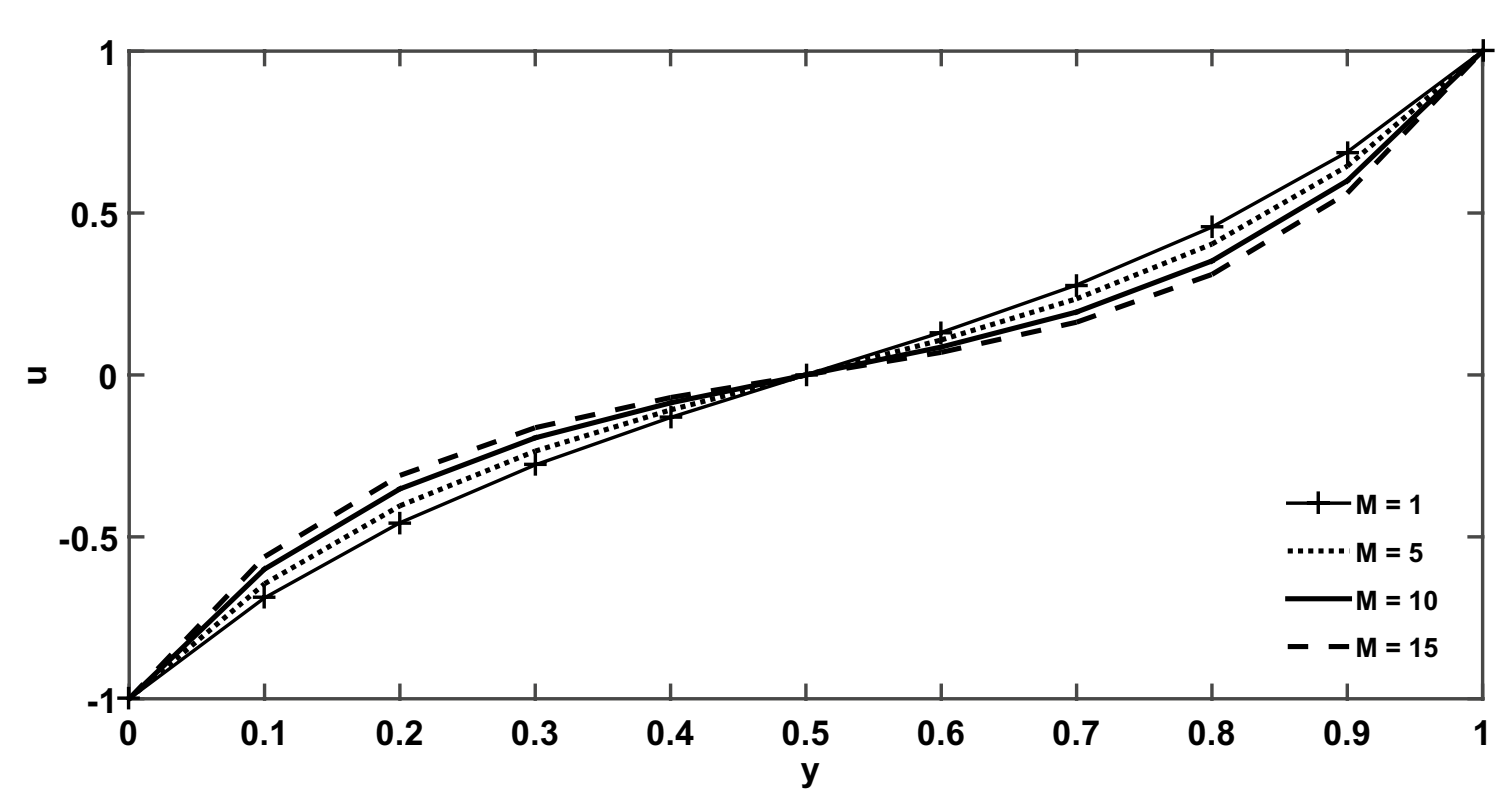

Fig. 2 Velocity portrait for diverse values of $M$, when $\phi=\pi / 3$ and $K=0.5$

\section{Results and discussion}

The current examination is a continuation work of Sukumar and Varma [12] with porous medium K, aligned angle $\phi$ and temperature gradient heat source instead of temperature dependent heat source and with different boundary condition $u^{*}=-u_{0}$ instead of slip condition $\frac{\partial u^{*}}{\partial y^{*}}=\frac{\alpha}{\sqrt{k}} u^{*}$ at $y^{*}=0$. The target of the current work is to contemplate the properties of temperature gradient heat source and entropy generation on aligned magnetic arena in the subsistence of thermal radiation, viscous dissipation and joules dissipation. The implications of physical parameters such as Brinkman number $\mathrm{Br}$, Hartmann magnetic number $M$, radiation parameter $N$, permeability parameter $\mathrm{K}$, aligned angle parameter $\phi$, characteristic temperature ratio $\omega$ and temperature gradient heat source parameter $Q$ are probed on the velocity and temperature distributions, Bejan number and entropy generation. The closed method result procured for velocity and temperature are selected to enumerate entropy generation. The conclusions are interpreted by graphs using MATLAB program. The mutations in profiles of velocity $u$ for diverse values of aligned angle parameter $\phi$ and Hartmann number $M$ are conferred in Figs. 2 and 3. It is perceived that the velocity of the fluid increased in lower part of the channel whereas it contracts in the upper part of the channel with rising $M$ or $\phi$. The consequences of Brinkman number $\mathrm{Br}$, Hartmann numbers $\mathrm{M}$ and temperature gradient-heat source $Q$ on temperature distribution are shown in Fig. 4. It was confirmed that the 


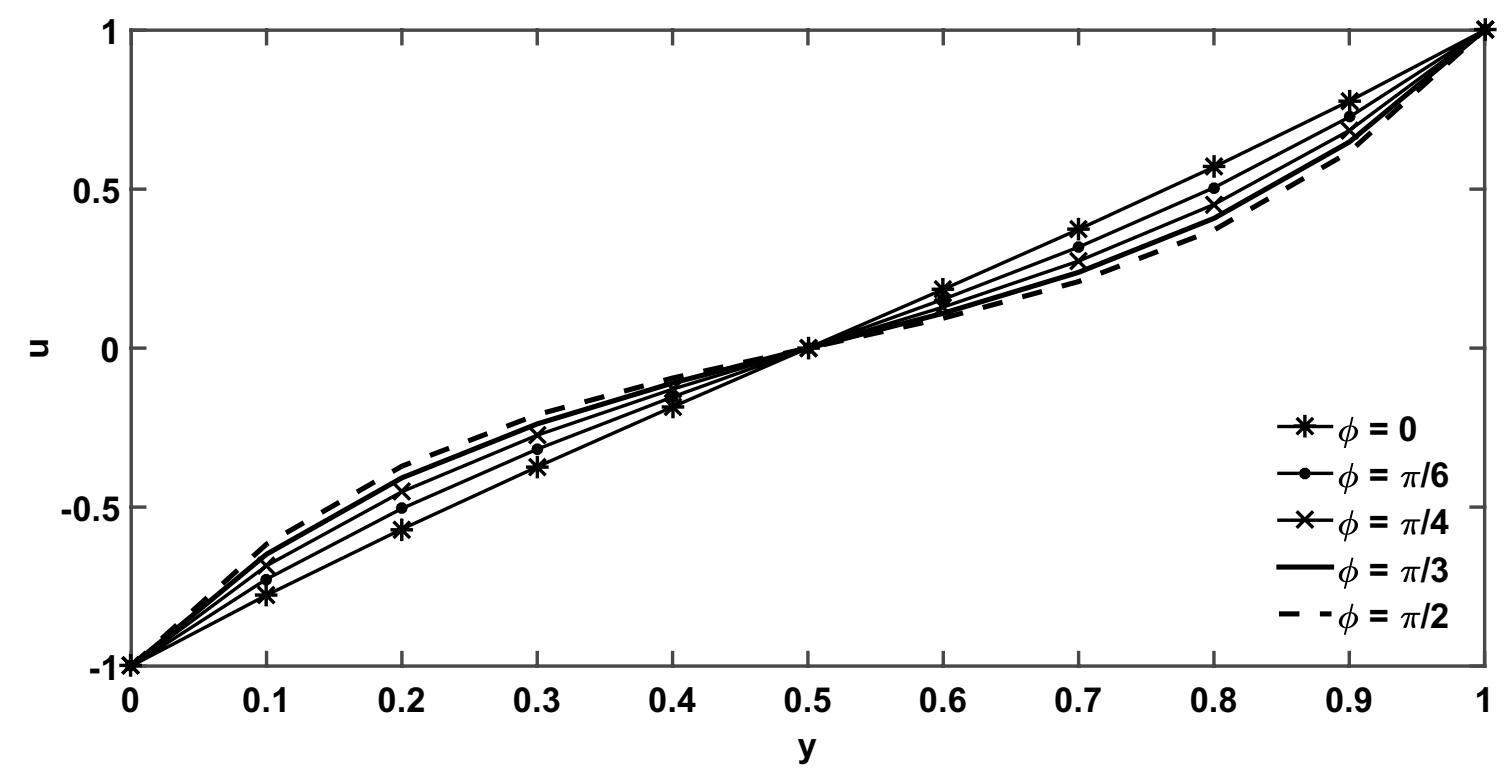

Fig. 3 Velocity portrait for diverse values of aligned angle parameter $\phi$ when $K=0.5$ and $M=10$

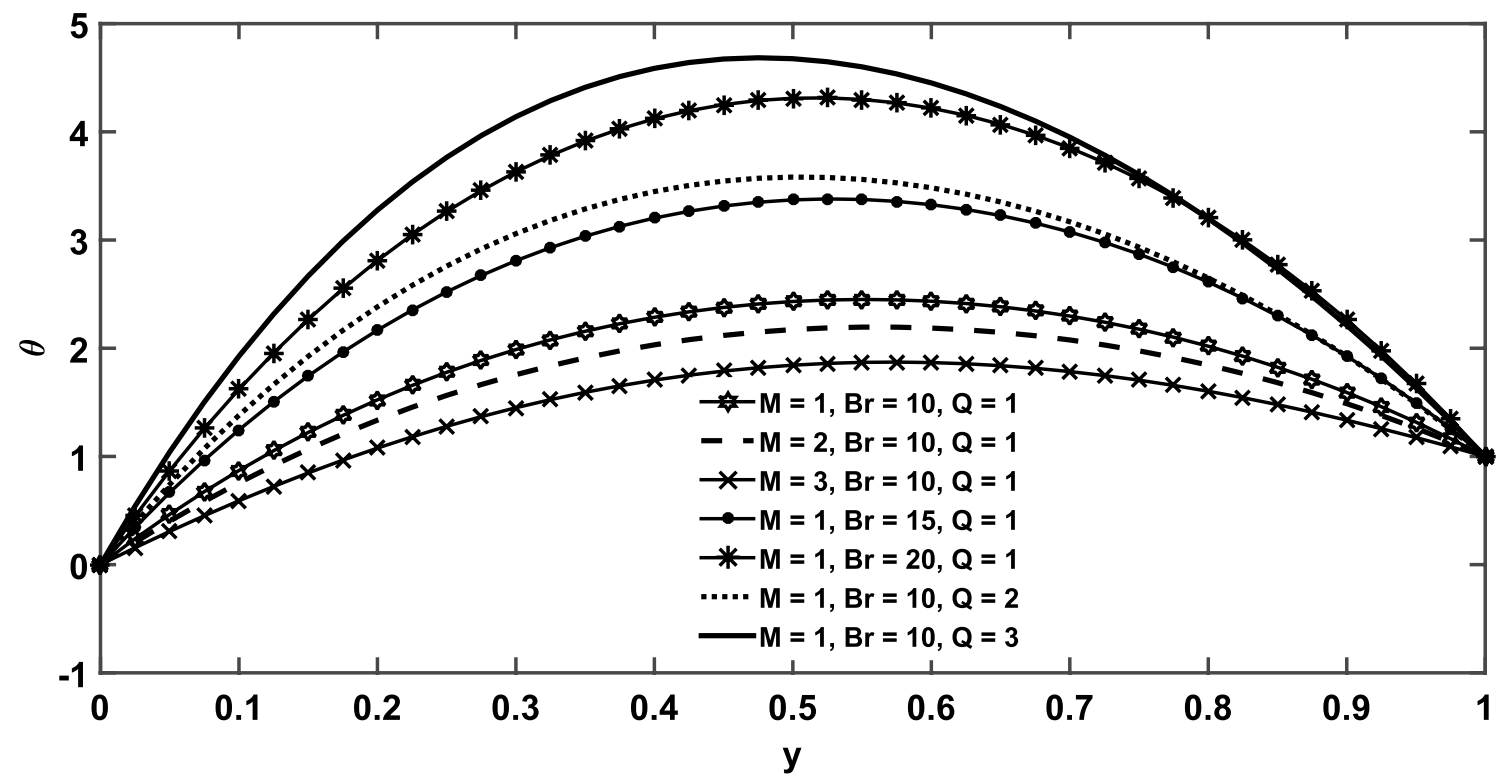

Fig. 4 Temperature portrait for diverse values of Hartmann and Brinkman numbers $\mathrm{Br}$ and $\mathrm{M}$ and gradient heat source parameter $\mathrm{Q}$ when $\mathrm{N}=1, \mathrm{~K}=1$ and $\phi=\pi / 3$

fluid temperature enriched with an escalation of temperature gradient heat source $Q$ and Brinkman number $B r$. The enhancement in Brinkman number repercussions in enhanced convective transport and $Q$ result is very much eloquent for the fluid flow model where transfer of heat is given prime imperative, whereas the mounting values of Hartmann number $M$ denigrate the fluid temperature.

Figure 5 emphasizes the changes of $\theta$-temperature distribution for diverse values of $N$ radiation parameter and aligned angle parameter $\phi$. It is noticed that the temperature distribution diminishes with intensifying $\mathrm{N}$ and $\phi$ values. This can be interpreted by the evidence that an escalation $N=\frac{4 \gamma^{*} T_{1}^{3}}{\alpha^{*} k}$ for $k$ and $T_{1}$ aids acceleration in the mean immersion constant $\alpha^{*}$. The deviation of radiative heat flux diminutions as $\alpha^{*}$ intensifications the rate of radiative heat transferred to the fluid and accordingly the fluid temperature decreases.

The diversity in entropy generation number Ns for conflicting values of aligned angle $\phi$, Hartmann magnetic 


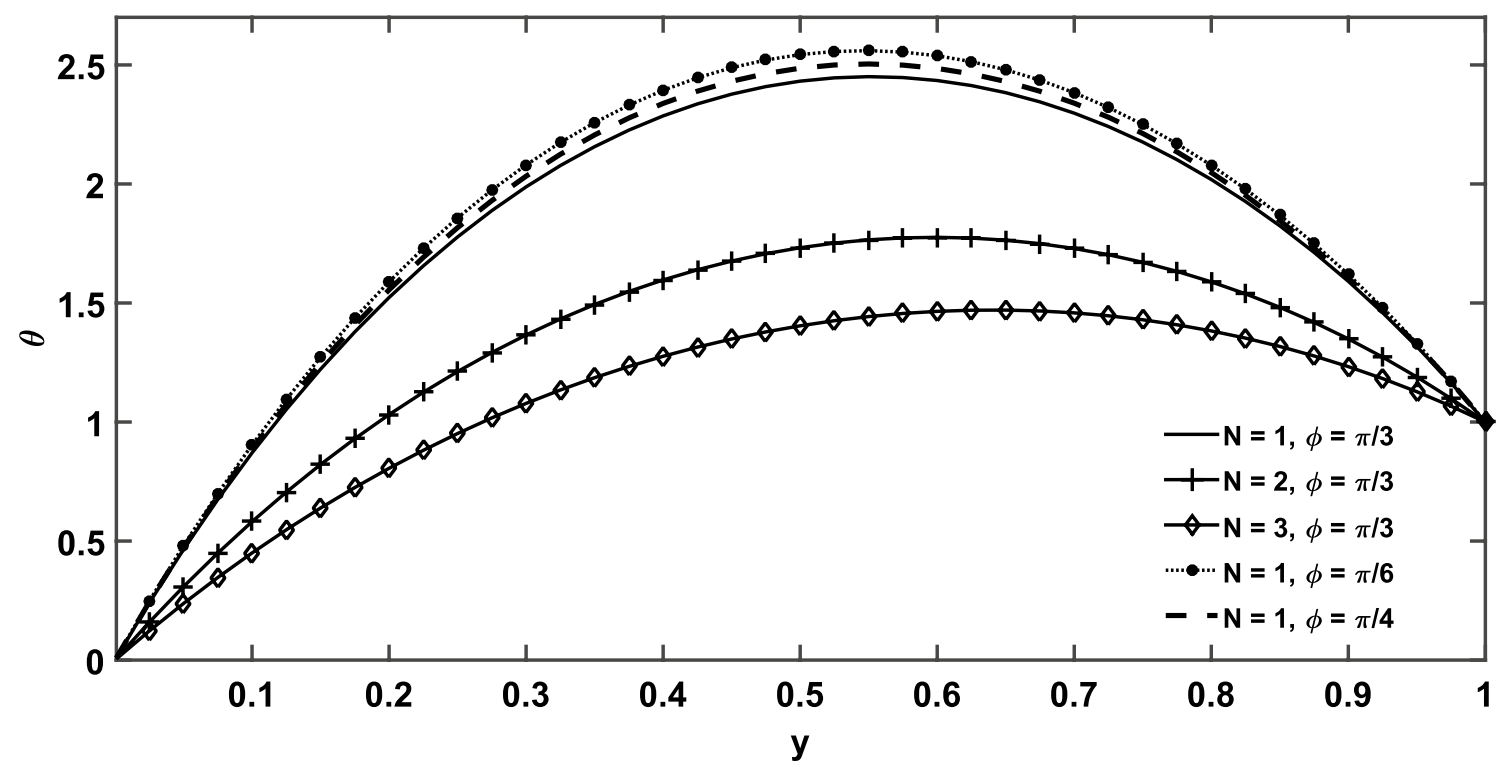

Fig. 5 Temperature portrait for diverse values of radiation parameter $\mathrm{N}$ and aligned angle parameter $\phi$ when $\mathrm{K}=1, \mathrm{Q}=1$ and $\mathrm{M}=1$

number $M$, Brinkman number $B r$, permeability parameter $K$ and temperature gradient heat source $Q$ are shown in Figs. 6, 7, 8. From Fig. 6 it is witnessed that in the channel the entropy generation accelerates with accelerating values of $M$ and $K$, this instigated by the transversely enforced hypnotic field retards the flow as well as Ohmic dissipation leads to intensification in heat in the channel with augmenting values of $\mathrm{M}$. Figure 7 demonstrates the impact of Brinkman number $\mathrm{Br}$ and temperature gradient heat source $\mathrm{Q}$ on the entropy generation. From the figure, it was endorsed that dissimilar enrichment values of the temperature gradient heat source lead to stimulating the entropy generation rate. But by Brinkman number the amount of entropy generation rises in the down part of the channel and decays in the upper part of the channel. It is highlighted that $\mathrm{Br}$ is a part of fluid erosion in the dissipative flow pattern. Figure 8 elucidates the effect of aligned angle parameter $\phi$ against $N_{s}$ entropy generation. It demonstrates that entropy accelerates in the conduit with escalating aligned angle. The difference in Bejan number Be for divergent values of flow parameters are exhibited in Figs. 9 and 10. Figure 9 demonstrates the influences of

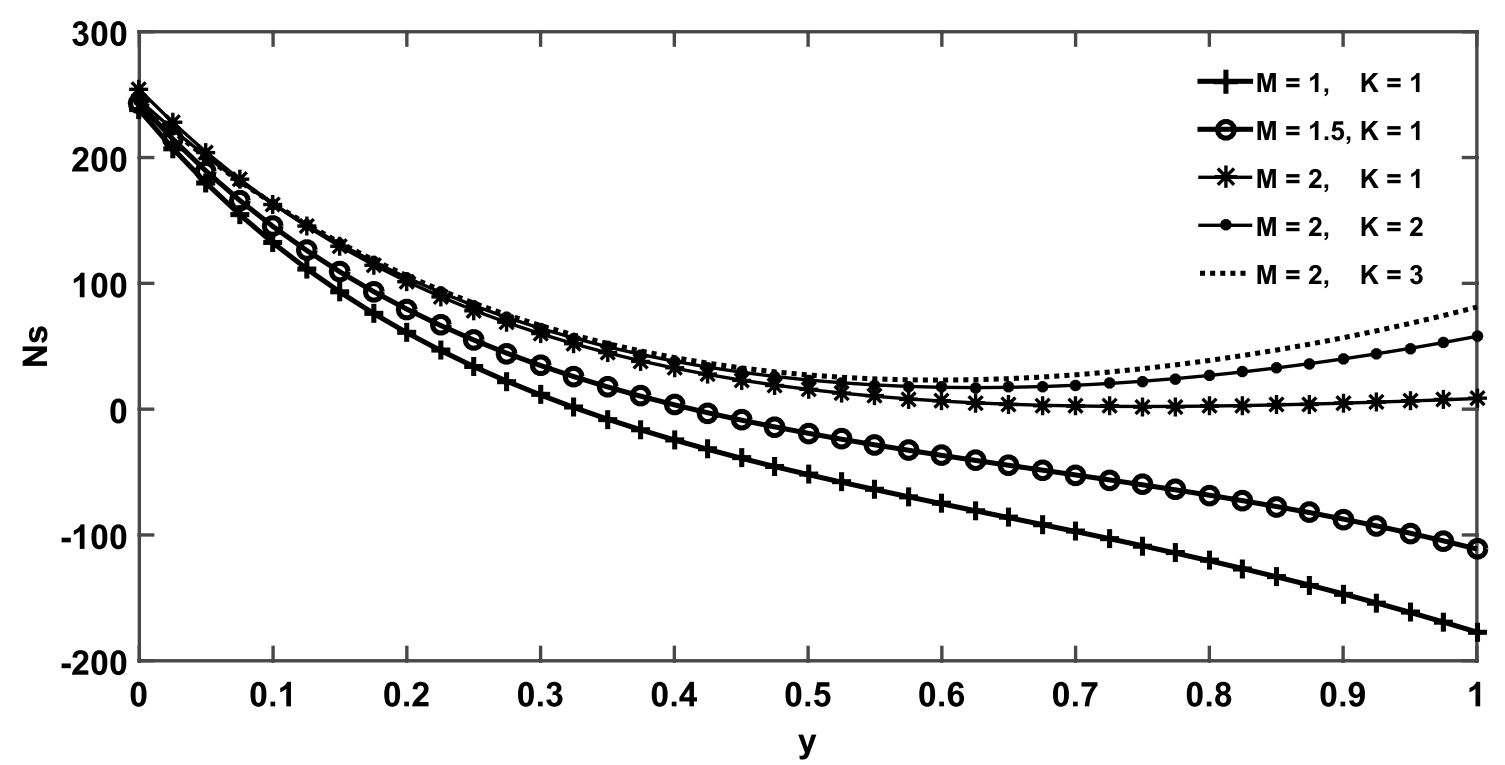

Fig. 6 Ns for diverse values of Hartmann number $M$ \& porosity parameter $K$ when $Q=1, N=1, \omega=0.4, \phi=\pi / 3$ and $B r=10$ 




Fig. $7 \mathrm{Ns}$ for diverse values of Brinkman number $\mathrm{Br}$ and temperature gradient heat source parameter $\mathrm{Q}$ when $\mathrm{M}=2, \mathrm{~K}=1, \mathrm{~N}=1, \phi=\pi / 3$ and $\omega=0.4$

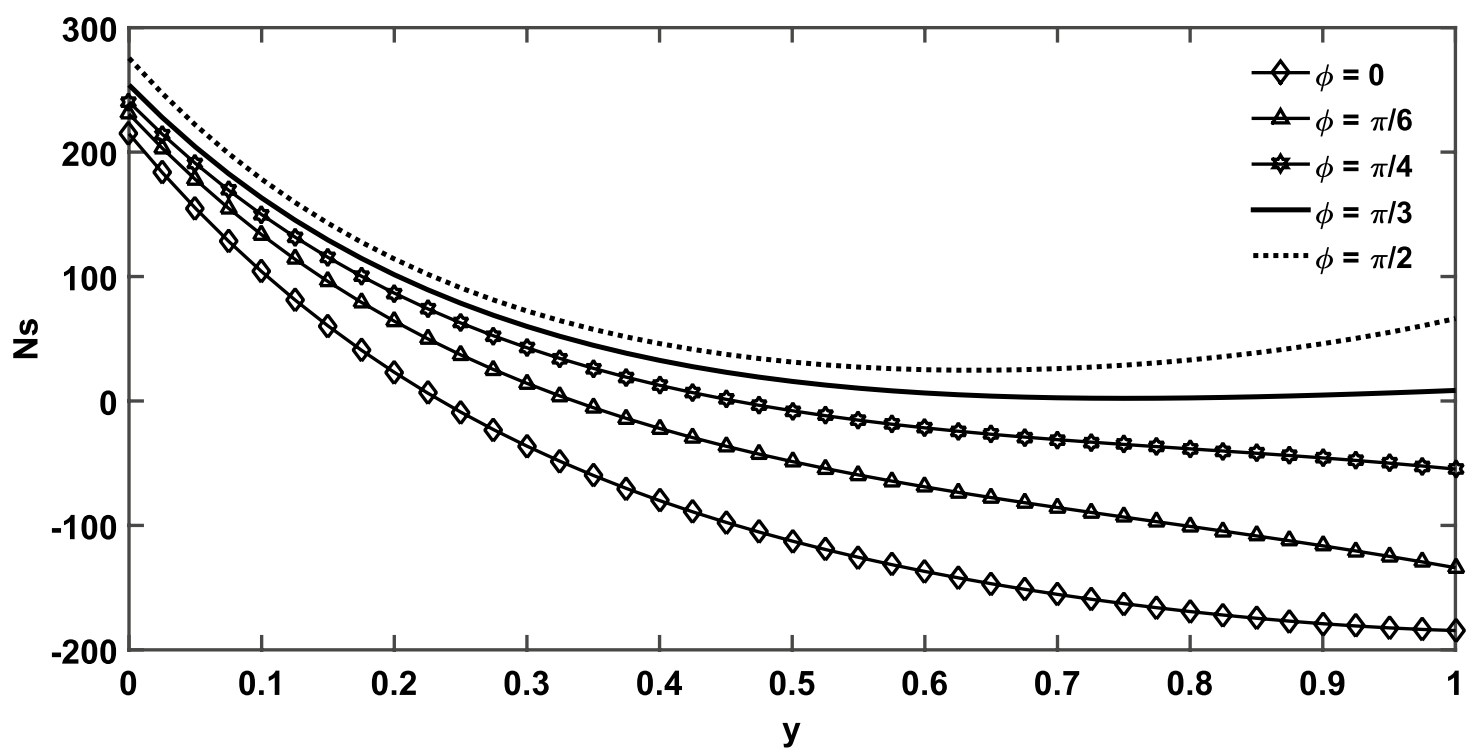

Fig. 8 Ns for diverse values of aligned angle parameter $\phi$ when $K=1, Q=1, N=1, M=1, \omega=0.4$ and $B r=10$

temperature gradient heat source $Q$ as well as radiation parameter $\mathrm{N}$ on Bejan number. The Bejan number raised by the incremental values of temperature gradient heat source parameter $\mathrm{Q}$ near lower and upper part of the channel while the slackening effect occurs in the middle of the channel. Opposite effect occurred in case of radiation parameter $\mathrm{N}$ i.e.: the Bejan number is diminished by means of the incremental values of radiation parameter $\mathrm{N}$ near lower and upper part of the channel while the intensification effect occurs in the middle region of the channel. Figure 10 depict that the Bejan number declined in the channel with increasing values of characteristic ratio $\omega$ and aligned angle parameter $\phi$.

Table 1 illustrates the shearing stress at the plates $y=$ zero $(0)$ and $y=$ one (1). From this table it is noticed that the intensifying values of $M$ and $\phi$ lead to raise shear stress at both plates. But conflicting influence was eventuated in case of porosity parameter K. Also we can see 


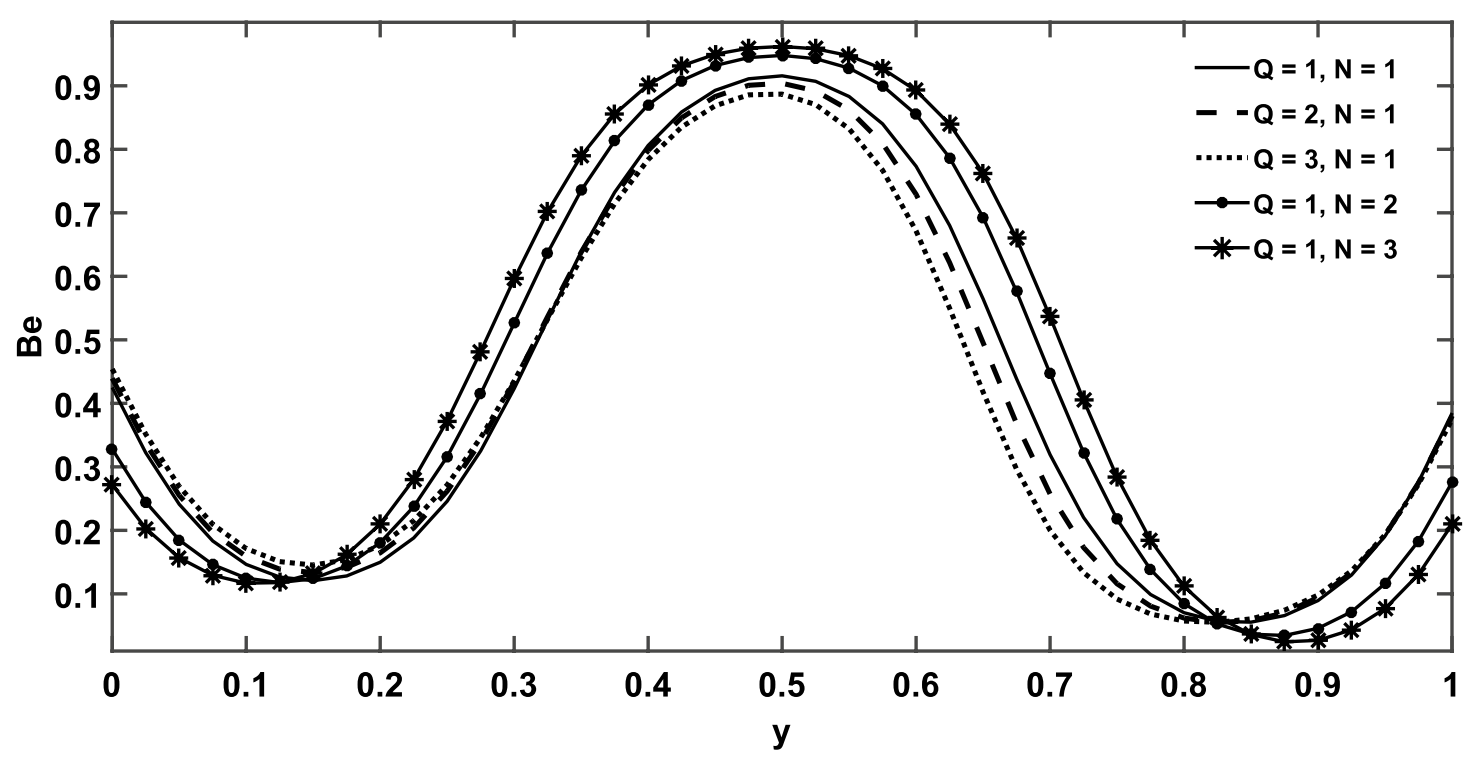

Fig. 9 Bejan number for diverse values of $\mathrm{N}$-radiation parameter and Q-temperature gradient heat source when $\mathrm{M}=2, \omega=0.4, \mathrm{Br}=10$, $\mathrm{K}=0.01$ and $\phi=\pi / 3$

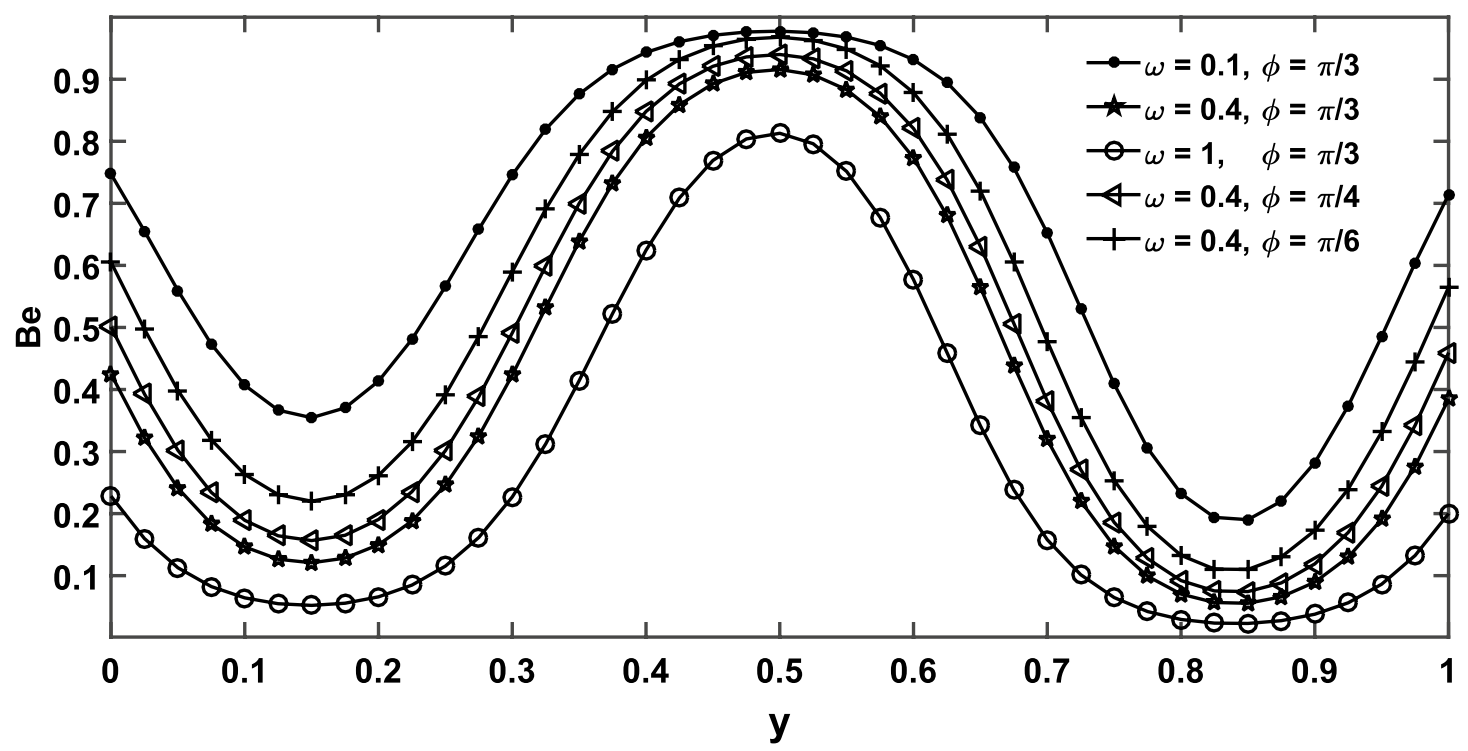

Fig. 10 Bejan number for diverse values of $\omega$-characteristic temperature ration and $\phi$-aligned angle parameter when $N=1, M=2, K=0.01$, and $\mathrm{Q}=1$

that shear stress is same at both plates (lower and upper plates). Table 2 represents the impacts of M-magnetic field parameter and K-permeability parameter on Nusselt number. It is perceived that the Nusselt number declines with an augment in $M$ or $\mathrm{K}$ at wall $\mathrm{y}=$ zero (0), whereas at wall $y=$ one (1), Nu decreases by increasing $M$ or K. Table 3 elucidates that Nusselt number lessening by an rise in Q-temperature gradient heat source parameter or Brinkman number $\mathrm{Br}$ at $\mathrm{y}=$ zero $(0)$ wall but reverse trend at the wall $y=$ one (1). Table 4 shows that with increasing aligned angle $\phi$ or radiation parameter $N$, the Nusselt number $\mathrm{Nu}$ accelerates at lower plate $\mathrm{y}=$ zero $(0)$ and decelerates at higher plate $y=$ one (1). 
Table 1 Skin friction at the plates $y=0$ and $y=1$

\begin{tabular}{lllll}
\hline $\mathrm{M}$ & $\mathrm{K}$ & $\phi$ & $\tau$ at $\mathrm{y}=0$ & $\tau$ at $\mathrm{y}=1$ \\
\hline 1 & 1 & $\pi / 3$ & 2.400278202397288 & 2.400278202397288 \\
2 & 1 & $\pi / 3$ & 2.626070570998663 & 2.626070570998663 \\
3 & 1 & $\pi / 3$ & 2.842313987807863 & 2.842313987807863 \\
1 & 2 & $\pi / 3$ & 2.322726139460428 & 2.322726139460427 \\
1 & 3 & $\pi / 3$ & 2.296608825340980 & 2.296608825340980 \\
1 & 1 & $\pi / 4$ & 2.322726139460428 & 2.322726139460427 \\
1 & 1 & $\pi / 6$ & 2.243965149218269 & 2.243965149218268 \\
\hline
\end{tabular}

\section{Conclusions}

Entropy generation in a horizontal channel entrenched with permeable medium in the existence of aligned magnetic field, temperature gradient heat source, thermal radiation and dissipations (viscous and joules) have been studied and analyzed. The results obtained from our examination are the velocity of the fluid escalated in lower part of the channel while it contracts in the upper part of the channel with intensifying magnetic field parameter $M$ and aligned magnetic field parameter $\phi$. Fluid temperature intensification with amplification of magnetic parameter $\mathrm{M}$ or temperature gradient heat source parameter $Q$. Brinkman number $\mathrm{Br}$ augments boundary layer density. Due to thermal radiation or aligned angle the thickness of thermal boundary layer becomes thinner. Ns escalates with intensifying values of $M$, aligned angle $\phi$, temperature gradient heat source parameter $Q$, characteristic temperature ration $\omega$ and permeability parameter $\mathrm{K}$. But contrast result is occurred in the case of $\mathrm{N}$ - radiation parameter. Entropy generation increases in lower part of the channel whereas the reversal effect is occurred in the upper part of the channel by Brinkman number $\mathrm{Br}$. When the magnetic parameter $\mathrm{M}$ accelerates the Bejan number diminished in the lower part of the channel and rises in the upper part channel. Bejan number will increase in lower and upper part of the channel with the cumulative values of $\mathrm{Br}$ whereas it decays in the middle region of the channel with the increasing values $\mathrm{Br}$. Reinforcement of characteristic temperature ration $\omega$ or aligned angle parameter $\phi$ leads to decelerate in Bejan number. Spurring the values of temperature gradient heat source $Q$ lead to Bejan number increases in lower \& higher divisions of the conduit but in the central segment of the channel the reversed trend is witnessed. Bejan number decreases at both lower and upper channel except in central region of the channel, the reversed trend is witnessed in middle region of the channel due to enhancement in diverse values of radiation parameter $N$. Increasing $M$ or $\phi$ the
Table 2 Nusselt number for diverse values of Hartmann number $M$ and porosity parameter $K$ with $B r=10$, $N=1, Q=1, \varphi=\frac{\pi}{3}$ and $y=0,1$

\begin{tabular}{|c|c|c|c|c|c|c|c|c|}
\hline \multirow[t]{2}{*}{$K \backslash M$} & \multicolumn{4}{|l|}{$-\theta^{1}(0)$} & \multicolumn{4}{|l|}{$-\theta^{1}(1)$} \\
\hline & 1 & 2 & 3 & 4 & 1 & 2 & 3 & 4 \\
\hline 1 & -9.9179 & -8.5343 & -6.3627 & -3.4933 & 6.7967 & 5.5688 & 3.6051 & 0.9863 \\
\hline 2 & -9.8949 & -8.4772 & -6.2645 & -3.3493 & 6.7586 & 5.5013 & 3.5018 & 0.8428 \\
\hline 3 & -9.8882 & -8.4588 & -6.2320 & -3.3012 & 6.7467 & 5.4793 & 3.4675 & 0.7949 \\
\hline 4 & -9.8851 & -8.4498 & -6.2158 & -3.2772 & 6.7409 & 5.4685 & 3.4505 & 0.7709 \\
\hline
\end{tabular}

\begin{tabular}{|c|c|c|c|c|c|c|c|c|}
\hline \multirow[t]{2}{*}{$B r \backslash Q$} & \multicolumn{4}{|l|}{$-\theta^{1}(0)$} & \multicolumn{4}{|l|}{$-\theta^{1}(1)$} \\
\hline & 1 & 2 & 3 & 4 & 1 & 2 & 3 & 4 \\
\hline 1 & -2.0984 & -2.9298 & -3.8491 & -4.8485 & -0.0412 & 0.4644 & 0.8822 & 1.2200 \\
\hline 5 & -5.5737 & -8.6929 & -12.1379 & -15.8790 & 2.9979 & 4.8498 & 6.3760 & 7.6060 \\
\hline 10 & -9.9179 & -15.8968 & -22.4988 & -29.6671 & 6.7967 & 10.3314 & 13.2432 & 15.5886 \\
\hline 15 & -14.2620 & -23.1007 & -32.8597 & -43.4553 & 10.5955 & 15.8131 & 20.1104 & 23.5712 \\
\hline
\end{tabular}

Table 3 Nusselt number for diverse values of brinkman number $B r$ and temperature gradient heat source parameter $Q$ with $N=1, M=1$, $K=1, \varphi=\frac{\pi}{3}$ and $y=0,1$

Table 4 Nusselt number for diverse values of aligned angle parameter $\phi$, radiation parameter $\mathrm{N}$ with $\mathrm{Br}=10, M=1$, $K=1, Q=1$ and $y=0,1$

\begin{tabular}{|c|c|c|c|c|c|c|c|c|}
\hline \multirow[t]{2}{*}{$\varphi \backslash N$} & \multicolumn{4}{|l|}{$-\theta^{1}(0)$} & \multicolumn{4}{|l|}{$-\theta^{1}(1)$} \\
\hline & 1 & 2 & 3 & 4 & 1 & 2 & 3 & 4 \\
\hline \multirow{4}{*}{$\begin{array}{l}\frac{\pi}{6} \\
\frac{\pi}{4} \\
\frac{\pi}{3} \\
\frac{\pi}{2}\end{array}$} & -10.2309 & -6.7381 & -5.1611 & -4.2637 & 7.0502 & 4.2591 & 2.9034 & 2.1030 \\
\hline & -10.0693 & -6.6387 & -5.0895 & -4.2077 & 6.9191 & 4.1721 & 2.8384 & 2.0512 \\
\hline & -9.9179 & -6.5457 & -5.0224 & -4.1553 & 6.7967 & 4.0909 & 2.7777 & 2.0027 \\
\hline & -9.7761 & -6.4586 & -4.9597 & -4.1063 & 6.6825 & 4.0150 & 2.7210 & 1.9574 \\
\hline
\end{tabular}


skin friction accelerates but decays by porosity $\mathrm{K}$ at both the lower and upper walls. As Brinkman number $\mathrm{Br}$ or temperature gradient heat source $Q$ rises, the Nusselt number decays at lower $(\mathrm{y}=0)$ wall and increases at upper $(y=1)$ wall.

The results that are obtained are helpful to study in what way to minimize or restrict energy wastages in the form of heat dissipation during energy generation, particularly in dealing with problems of heat transfer. This flow model can be extended to study for non-Newtonian fluid flows.

Acknowledgements The authors thank their respective Departments of Mathematics at RVR \& JC College of Engineering, Guntur, DNR College of Engineering and Technology, Bhimavaram and PB Siddhartha College of Arts and Science, Vijayawada. Also authors are thankful to the reviewers for their constructive comments to improve the paper significantly.

\section{Declarations}

Conflict of interest The authors declare that they have no conflict of interest.

Open Access This article is licensed under a Creative Commons Attribution 4.0 International License, which permits use, sharing, adaptation, distribution and reproduction in any medium or format, as long as you give appropriate credit to the original author(s) and the source, provide a link to the Creative Commons licence, and indicate if changes were made. The images or other third party material in this article are included in the article's Creative Commons licence, unless indicated otherwise in a credit line to the material. If material is not included in the article's Creative Commons licence and your intended use is not permitted by statutory regulation or exceeds the permitted use, you will need to obtain permission directly from the copyright holder. To view a copy of this licence, visit http://creativecommons.org/licenses/by/4.0/.

\section{Appendix}

$$
\begin{aligned}
c_{1} & =\frac{1+e^{-M_{1}}}{e^{M_{1}}-e^{-M_{1}}} \\
c_{2} & =-1-c_{1} \\
c_{3} & =-c_{4}-c_{5}-c_{6} \\
c_{4} & =\frac{c_{5}\left(e^{2 M_{1}}-1\right)+c_{6}\left(e^{-2 M_{1}}-1\right)+c_{7}-1}{1-e^{-\frac{Q}{B}}} \\
c_{5} & =\frac{B r c_{1}^{2}\left(M^{2} \sin ^{2} \phi-M_{1}^{2}\right)}{\left(4 B M_{1}^{2}+2 M_{1} Q\right)} \\
c_{6} & =\frac{B r c_{2}^{2}\left(M^{2} \sin ^{2} \phi-M_{1}^{2}\right)}{\left(4 B M_{1}^{2}-2 M_{1} Q\right)} \\
B & =1+\frac{4 N}{3} \\
M_{1}^{2}= & M^{2} \sin ^{2} \phi+\frac{1}{K}
\end{aligned}
$$

\section{References}

1. Bejan A (1994) Entropy generation through heat and fluid flow. Wiley, New Jersey

2. Bejan A (1996) Entropy generation minimization. CRC Press, Boca Raton

3. Rosen MA (1999) Second-law analysis: approaches and implications. Int J Energy Res 23(5):415-429

4. Narusawa U (2001) The second-law analysis of mixed convection in rectangular ducts. Heat Mass Transf 37:197-203

5. Ko TH, Cheng CH (2007) Numerical investigation on developing laminar forced convection and entropy generation in a wavy channel. Int Commun Heat Mass Transf 34(8):924-933

6. Delavar MA, Hedayatpour M (2012) Forced convection and entropy generation inside a channel with a heat-generating porous block. Heat Transf Asian Res 41(7):580-600

7. Makinde OD, Eegunjobi AS (2013) Entropy generation in a couple stress fluid flow through a vertical channel filled with saturated porous media. Entropy 15(11):4589-4606

8. Chinyoka T, Makinde OD (2013) Analysis of entropy generation rate in an unsteady porous channel flow with Navier slip and convective cooling. Entropy 15(6):2081-2099

9. Das S, Jana RN (2014) Entropy generation due to MHD flow in a porous channel with Navier slip. Ain Shams Eng J 5(2):575-584

10. Hassan AR, Gbadeyan JA (2015) A reactive hydro magnetic internal heat generating fluid flow through a channel. Int J Heat Technol 3(3):43-50

11. Hatami M, Song D, Jing D (2016) Optimization of a circular wavy cavity filled by nano fluid under the natural convection heat transfer condition. Int J Heat Mass Transf 98:758-767

12. Sukumar M, Varma SVK (2016) Entropy generation and temperature dependent heat source effects on MHD Couette flow with permeable base in the presence of radiation and viscous dissipation. Middle East J Sci Res 24(8):2577-2588

13. Bhattacharyya S, Chattopadhyay H, Swami A, Uddin MK (2016) Convective heat transfer enhancement and entropy generation of laminar flow of water through a wavy channel. Int J Heat Technol 35(3):433-446

14. Rashidi MM, Bagheri S, Momoniat E, Freidoonimehr N (2017) Entropy analysis of convective MHD flow of third grade nonNewtonian fluid over a stretching sheet. Ain Shams Eng J 8(1):77-85

15. Baag S, Mishra SR, Dash GC, Acharya MR (2017) Entropy generation analysis for viscoelastic MHD flow over a stretching sheet embedded in a porous medium. Ain Shams Eng J 8(4):623-632

16. Abiodun OA, Onija TU (2017) Entropy generation and irreversibility analysis due to steady mixed convection flow in a vertical porous channel. Int J Heat Technol 35(3):433-446

17. Wenhui Tanga M, Hatami M, Zhoua J, Jinga D (2017) Natural convection heat transfer in a nano fluid filled cavity with double sinusoidal wavy walls of various phase deviations. Int J Heat Mass Transf 115:430-440

18. Rashidi S, Esfahani JA, Maskaniyan M (2017) Applications of magnetohydrodynamics in biological systems-a review on the numerical studies. J Magn Magn Mater 439:358-372

19. Rashidi S, Mahian O, Languri EM (2018) Applications of nanofluids in condensing and evapoarating systems. J Therm Anal Calorim 131:2027-2039

20. Srinivasacharya D, Hima Bindu K (2018) Entropy generation due to micro polar fluid flow between concentric cylinders with slip and convective boundary conditions. Ain Shams Eng J 9(2):245-255

21. Sudhakar M, Balamurugan KS (2018) Entropy generation analysis in a vertical porous channel with Navier slip in the 
presence of viscous dissipation and heat source. Int J Mech Prod Eng Res Dev 8(5):261-270

22. Balamurugan KS, Sudhakar M, Raju VCC (2018) Entropy generation and dissipative effects on Couette flow in an aligned magnetic field with radiation and heat source. Int. J. Eng. Sci. Invention 7(4):1-11

23. Tlau L, Ontela S (2020) Second law analysis for mixed convection nanofluid flow in an inclined channel with convectively heated walls. Heat Transf 49(2):1035-1064

24. Pavan Kumar Reddy M, Ramana Murthy JV (2019) Entropy analysis for heat transfer in a rectangular channel with suction. Heat Transf Asian Res 48(7):2773-2798
25. Naz R, Noor M, Shah Z, Sohail M, Kumam P, Thounthong P (2020) Entropy generation optimization in MHD pseudoplastic fluid comprising motile microorganisms with stratification effect. AEJ 59(1):485-496

Publisher's Note Springer Nature remains neutral with regard to jurisdictional claims in published maps and institutional affiliations. 